Cahiers d'études africaines

$240 \mid 2020$

Soudan : identités en tension

SALOMON Noah. - For Love of the Prophet

Lucie Revilla

(2) OpenEdition

Journals

Édition électronique

URL : https://journals.openedition.org/etudesafricaines/32958

DOI : 10.4000/etudesafricaines.32958

ISSN : 1777-5353

Éditeur

Éditions de l'EHESS

Édition imprimée

Date de publication : 2 décembre 2020

Pagination : 1040-1042

ISSN : 0008-0055

Référence électronique

Lucie Revilla, « Salomon Noah. - For Love of the Prophet », Cahiers d'études africaines [En ligne], 240 |

2020, mis en ligne le 02 décembre 2020, consulté le 06 janvier 2023. URL : http://

journals.openedition.org/etudesafricaines/32958; DOI : https://doi.org/10.4000/etudesafricaines. 32958

Ce document a été généré automatiquement le 6 janvier 2023.

Tous droits réservés 


\title{
SALOMON Noah. - For Love of the Prophet
}

\author{
Lucie Revilla
}

\section{RÉFÉRENCE}

SALOMOn Noah. - For Love of the Prophet. Princeton, Princeton University Press, 2016, 264 p., bibl., index, ill.

1 L'ouvrage de Noah Salomon renouvelle sensiblement les approches du politique sur l'Inqādh (1989-2019), période caractérisée par la tentative d'imposition d'un «État islamique " ("al dawla al islāmiyya») menée par le mouvement islamiste soudanais. L'auteur innove autant par l'approche choisie que par les objets explorés. Dans une démarche qui rappelle fortement celle d'A. Gramsci, que l'on peut reprocher à l'auteur de ne pas citer, le pouvoir est producteur de représentations véhiculant l'idéologie dominante bien au-delà des institutions étatiques. Cette hégémonie est susceptible d'appropriations et de reformulations multiples par les individus qui y sont soumis. Dans la droite lignée des travaux s'inspirant de M. Foucault, la proposition de $\mathrm{N}$. Salomon est d'analyser les formes de subjectivation que le régime islamiste a produites. Au-delà de la vision coercitive du pouvoir, celui-ci est investi de sens par les individus, rendant alors difficile la stricte séparation entre État et société. À rebours des recherches en science politique qui discutent du projet islamiste soudanais à l'aune de son échec ou de sa réussite, cet ouvrage décrit des formes diffuses de pouvoir et leurs effets sur les subjectivités. Cependant, l'auteur entretient une certaine confusion entre l'analyse de l'État et celle de la culture politique. Tandis que c'est bien sur cette seconde notion que $\mathrm{N}$. Salomon reste le plus stimulant, le cadre théorique tend à donner une conception extensive de l'État au point qu'il devient difficile de comprendre ce qui n'en relève pas. Cette critique ne doit pas éclipser l'apport fondamental que N.Salomon offre sur l'articulation entre idéologie et pratiques sociales. 
2 Dans le premier chapitre, l'auteur montre que le régime islamiste a érigé la référence religieuse en mode de gouvernement, là où l'administration coloniale avait entamé le même projet. Il revient sur la création d'institutions destinées à " civiliser ", et donc à contraindre, des modes d'expression religieuse perçus comme potentiellement déstabilisateurs, car attachés aux confréries soufies tenues pour responsables de la mobilisation mahdiste à la fin du XIXe siècle. Prolongeant les recherches de T. Asad dans ce domaine, N.Salomon explique comment l'administration coloniale a transformé une "pratique privée en religion publique» (p.35). Ce basculement institutionnalise une forme d'orthodoxie là où l'interprétation de la référence religieuse restait labile et l'adaptabilité à la situation, la règle. En réintroduisant le référent religieux au sein des institutions, l'Inqādh se trouverait dans la continuité du projet colonial, dépendante d'une vision occidentale que le mouvement islamiste a pourtant tant dénoncée. Si la démonstration semble convaincante, N. Salomon ne donne qu'un aperçu trop bref des conceptions endogènes du réformisme islamique et des influences antérieures à la colonisation qui contribuent à donner un contour légaliste à l'application de la règle religieuse ${ }^{1}$.

3 Le second chapitre porte sur "l'architecture de l'islamisme tardif» (p.56) et les multiples défis auxquels le mouvement est confronté, à commencer par les accords de paix de 2005 qui poussent le régime de Khartoum à des compromis jusque-là jamais envisagés ${ }^{2}$. Contrairement aux tenants du post-islamisme, N. Salomon estime que la séparation du Sud-Soudan est un levier de réaffirmation du projet islamiste et non pas "la marque de son échec» (p.60)3. Le public appréciera la mobilisation de textes d'intellectuels islamistes traduits et utilisés dans la démonstration. L'analyse du "projet civilisationnel » ("al mashrū' al hadăāì), promu par le régime comme modèle de réforme morale, s'est surtout intéressée au domaine politique et institutionnel, laissant dans l'ombre ses effets dans la sphère culturelle. Or, cette période est marquée par le développement de nouveaux modes de production de la connaissance et de l'art.

4 Les chapitres 3 et 4 traitent de ces deux formes culturelles aux effets politiques fondamentaux, et c'est là que l'analyse de N. Salomon est la plus percutante. C'est d'abord par un mode épistémologique rationalisant que le régime de l'Inqādh a objectivé son projet moral de transformation de l'État et de l'individu. Les années 1990-2000 sont un moment de relative démocratisation de l'enseignement supérieur. La création d'universités sous contrôle islamiste s'inscrit dans une ambition bien plus large que la seule stratégie clientéliste de cooptation par la fourniture de services publics et charrie avec elle un véritable projet politique. C'est la relation hiérarchique essentielle à la transmission du savoir qui est réformée en remettant en cause des modes antérieurs d'acquisition de la connaissance. Alors que le régime soufi de révélation est fondé sur la persistance d'un domaine du «secret» et justifie la médiation d'une élite spirituelle, le régime de connaissance promu par le mouvement islamiste repose sur des méthodes didactiques. Ce n'est plus l'appartenance à une ascendance spirituelle qui fonde l'autorité politique, mais un raisonnement perçu comme rationnel. La «fondamentalisation du savoir» ("tâ’șil ») dote le projet intellectuel islamiste d'une prétention égalitaire, l'accès à la connaissance et donc au pouvoir devient (théoriquement) accessible à tou.te.s sans l'intermédiation d'une autorité spirituelle.

5 Le chapitre 4 parlera à tou.te.s les auditeur.trice.s de radio au Soudan, avec l'analyse fouillée de la station al-Kawthar ${ }^{4}$ qui réinvente la diffusion du madịh. Cette forme 
classique de poésie soufie est réappropriée par le régime, qui transforme «l'expérience esthétique " en véritable "technologie de pouvoir", destinée à cultiver la piété religieuse autour de l'adoration du Prophète. Les postures inhérentes à l'écoute radiophonique diffèrent des registres d'énonciation soufie. Basées sur un effort personnel de concentration, elles transforment l'exercice individuel de la prière en " aspiration collective " au service de la fabrication de «nouveaux imaginaires de la nation» (p.133). La pratique rituelle se fait alors véritable répertoire de mobilisation morale en faveur du projet étatique.

Le dernier chapitre revient sur une figure qui incarne à elle seule l'impossible monopolisation par l'État de l'énonciation du religieux et du politique. Sheikh al Bura'i, chef spirituel issu de la confrérie Sammāniyya, a marqué les années 1990-2000. Revêtant un apolitisme déclaré, il n'en est pas moins une figure politique de premier ordre par sa version d'un islam populaire et du potentiel de résistance au projet intellectuel islamiste qu'il incarne. Jamais réellement coopté par le régime, mais jamais ouvertement opposé à l'État, al Bura'i illustre les ambivalences de nombreux individus vis-à-vis de l'Inqā $d h$. Indispensable par l'amplitude de l'audience qu'il touchait, al Bura'i véhiculait pourtant des catégories d'entendement radicalement divergentes du régime, poussant malgré tout ce dernier à en reconnaître la puissance d'évocation ${ }^{5}$.

7 L'ouvrage de N. Salomon est un appel vivifiant à réinvestir l'étude des pratiques et confréries soufies, afin de se défaire d'une routinisation de l'usage des catégories dichotomiques du politique et du religieux, et à en saisir les définitions mouvantes audelà des seules organisations partisanes ou des institutions étatiques.

\section{NOTES}

1. Voir A. El AfFendI, Turabi's Revolution: Islam and Power in the Sudan, Londres, Grey Seal, 1991, pp. 8-24, notamment concernant l'influence des courants réformistes des XVIII ${ }^{\mathrm{e}}$ et XIX ${ }^{\mathrm{e}}$ siècles et des mouvements néo-soufis.

2. L'auteur reprend ici les conclusions d'E. A HMED, que l'on peut retrouver dans "L'Islam politique au Soudan. Les islamistes à l'épreuve de la direction de l'État (1989-2004) », in R. OTAYEK \& B. SOARES (dir.), Islam, État et société en Afrique, Paris, Karthala, pp. 293-326.

3. C'est ce que W. BERRIDGE souligne elle aussi: voir "The Ambiguous Role of the Popular, Society and Public Order Police in Sudan, 1983-2011 ", Middle Eastern Studies, 49 (4), 2013, pp. 528-546.

4. Nom d'un fleuve du paradis dans le texte coranique.

5. L'anecdote sur la rencontre entre al Bura'i et Al-Tourabi est à ce titre saisissante. 


\section{AUTEURS}

\section{LUCIE REVILLA}

Laboratoire des Afriques dans le monde (LAM), Sciences Po Bordeaux, France 